\title{
Elastic Constants, Optical Phonons, and Molecular Relaxations in the High Temperature Plastic Phase of the $\mathrm{CH}_{3} \mathrm{NH}_{3} \mathrm{PbBr}_{3}$ Hybrid Perovskite
}

Antoine Létoublon, ${ }^{* \dagger}$ ' Serge Paofai, ${ }^{\ddagger}$ Benoît Rufflé, ${ }^{\S}$ Philippe Bourges, ${ }^{\|}$Bernard Hehlen, ${ }^{\S}$ Thierry Michel, ${ }^{\S}$ Claude Ecolivet, ${ }^{\perp}$ Olivier Durand, ${ }^{\dagger}$ Stéphane Cordier, ${ }^{\ddagger}$ Claudine Katan, ${ }^{\ddagger}$ and Jacky Even ${ }^{*}, \dagger$

${ }^{\dagger}$ UMR FOTON, CNRS, INSA-Rennes, F- 35708 Rennes, France

${ }^{\ddagger}$ Institut des Sciences Chimiques de Rennes, UMR 6226, CNRS, Université de Rennes 1, F-35042 Rennes, France

${ }^{\S}$ Laboratoire Charles Coulomb (L2C), UMR 5221 CNRS-Université de Montpellier, F-34095 Montpellier, France

"LLB, CEA, CNRS, Université Paris-Saclay, CEA Saclay, F-91191 Gif-sur-Yvette, France

${ }^{\perp}$ Institut de Physique de Rennes, UMR 6251, CNRS, Université de Rennes 1, F-35042 Rennes, France

\section{Supporting Information}

ABSTRACT: Low frequency dynamics has been studied in a $\mathrm{CH}_{3} \mathrm{NH}_{3} \mathrm{PbBr}_{3}$ hybrid perovskite single crystal by using four different spectroscopy techniques: coherent inelastic neutron, Raman and Brillouin scatterings, and ultrasound measurements. Sound velocities were measured over five decades in energy to yield the complete set of elastic constants in a hybrid halide perovskite crystal in the pseudocubic plastic phase. The $\mathrm{C}_{44}$ shear elastic constant is very small, leading to a particularly low resistance to shear stress. Brillouin scattering has been used to study the relaxation dynamics of methylammonium

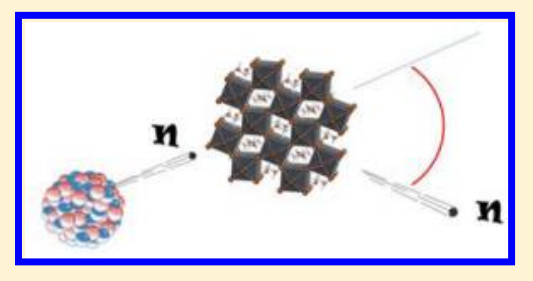
cations and to evidence translation-rotation coupling associated with the cubic to tetragonal phase transition at $T_{\mathrm{c}} \approx 230 \mathrm{~K}$. Low frequency and highly damped optical phonons observed using both Raman and inelastic neutron below $18 \mathrm{meV}$, do not present softening close to $T_{\mathrm{c}}$. The critical dynamics at $T_{\mathrm{c}} \approx 230 \mathrm{~K}$ is compatible with an order-disorder character, dominated by relaxational motions of the molecules.

$\mathrm{D}$ uring the past few years, hybrid halide perovskites have been intensively studied as promising materials for photovoltaic and optoelectronic applications. ${ }^{1-8}$ Though most experimental studies have been performed on $\mathrm{MAPbX}_{3}$ compounds, where $\mathrm{MA}=\mathrm{CH}_{3} \mathrm{NH}_{3}{ }^{+}$is the methylammonium cation and $\mathrm{X}=\mathrm{I}, \mathrm{Br}$, or $\mathrm{Cl}$ an halogen atom, the halide perovskite bulk and alloy compounds are nowadays also based on the formamidinium $\left(\mathrm{FA}=\mathrm{HC}\left(\mathrm{NH}_{2}\right)_{2}^{+}\right)$and cesium $\left(\mathrm{Cs}^{+}\right)$ cations to enhance photoconversion or light emission efficiencies. ${ }^{9-11}$ However, optical absorption and charge carrier mobilities are among the stumbling blocks that limit the maximum photoconversion of solar cells. Previous experimental studies have successfully evidenced the influence of the structural properties on the optoelectronic properties of the hybrid halide perovskites, but a comprehensive understanding is still lacking. ${ }^{12-14}$ Besides a few older experimental studies dedicated to the dynamics of the MA cation, ${ }^{15-17}$ a number of experimental and theoretical papers appeared recently addressing the structural and vibrational properties of $\mathrm{MAPbX}_{3}$ compounds. ${ }^{18-27}$ Despite the importance of the macroscopic mechanical properties for material processing, thermal and photostabilities or at the microscopic level for inelastic carrier scattering or exciton screening, ${ }^{13,28}$ to the best of our knowledge very few experimental studies have been reported so far on the acoustic phonons. ${ }^{29,30}$ The technologically important isostructural $\mathrm{MAPbI}_{3}$ and $\mathrm{MAPbBr}_{3}$ hybrid perovskite compounds have a primitive cubic structure at high temperature and undergo a cubic $(P m-3 m)$ to tetragonal (I4/ $\mathrm{mcm})$ antiferrodistortive phase transition ${ }^{15,31-33}$ respectively at $T_{\mathrm{c}} \sim 333 \mathrm{~K}$ and $T_{\mathrm{c}} \sim 230 \mathrm{~K}(227 \mathrm{~K}$ to $233 \mathrm{~K}$ according to different experimental studies ${ }^{15,17,31,34}$ and $231 \mathrm{~K}$ in this work). Recent detailed structural X-ray and neutron diffraction investigations indicate highly anisotropic displacement ellipsoids of the halogen ions. ${ }^{34}$ It may correspond to a large damping of low-frequency optical modes associated with inphase and antiphase rotations of the $\mathrm{PbX}_{6}$ octahedra. ${ }^{35-37}$ This behavior was already observed in the $\mathrm{CsPCl}_{3}$ bulk crystal by neutron scattering. ${ }^{37}$ It is also unclear whether this strong anharmonicity is at the origin of the structural $P m-3 m$ to I4/ $\mathrm{mcm}$ phase transition or not. A displacive character of this structural transition would correspond to an anomalous behavior of the frequency and damping of the critical optical phonon mode at the phase transition temperature $T_{c}$. For instance, the so-called "lattice melting" phenomenon observed around the ferroelastic structural phase transition of $\mathrm{Na}_{2} \mathrm{CO}_{3}{ }^{38}$ is also related to a strong anharmonicity of atomic displacements. It leads to large lattice strain and vanishing elastic constants at $T_{\mathrm{c}}$. Although the same behavior is not expected at $T_{\mathrm{c}}$ in $\mathrm{MAPbX}_{3}$ compounds because the $\mathrm{Pm}-3 \mathrm{~m}$ to $I 4 / \mathrm{mcm}$

Received: August 1, 2016

Accepted: September 7, 2016

Published: September 7, 2016 
phase transition is not ferroelastic, a linear-quadratic coupling between the transition order-parameter and the strain was predicted. $^{36}$ This may partially affect the elastic constants.

The stochastic reorientational motion of the MA cation at high temperature has been studied by various experimental and theoretical approaches. ${ }^{36,39-41}$ Theory predicts linear translation-rotation coupling that leads to a softening of the perovskite lattice as well as a damping of the sound waves propagating in the crystal. ${ }^{36}$ Correlated tumbling of the MA cations are also expected to play a role in the mechanism of the $P m-3 m$ to $I 4 / \mathrm{mcm}$ phase transition. A pseudospin variable related to such stochastic motions may be related to the order parameter and rather suggests an order-disorder character of the structural instability at $T_{\mathrm{c}}{ }^{36}$ Moreover, analogy with classical plastic crystals at high temperature has prompted the prediction of an orientational glassy state at low temperature. ${ }^{36}$ Indeed, recent experimental investigations combining dielectric measurements and calorimetry are consistent with this hypothesis. $^{42}$

The aim of this work is to provide a detailed analysis of low energy structural excitations in the high temperature plastic phase, by combining coherent neutron, Raman, and Brillouin scattering (BS), as well as ultrasonic (US) measurements. Using large single crystals of $\mathrm{MAPbBr}_{3}$ offers the unique opportunity to investigate the structural instabilities of the $\mathrm{MAPbX}_{3}$ family. In fact, it provides a RT high symmetric cubic $P m-3 m$ phase, avoiding coexistence of ferroelastic domains and crystal twinning. $\mathrm{MAPbBr}_{3}$ is also particularly suited to common laser sources used for Raman and Brillouin optical spectroscopies dedicated to vibrational properties, thus preventing resonant excitations. Overall, the experimental study shows that strong anharmonicity as well as disordered reorientational motions of the methylammonium cations dominate the spectrum of low energy structural excitations in $\mathrm{MAPbX}_{3}$ compounds. The results are consistent with an order-disorder character of the cubic to tetragonal structural phase transition, which affects the elastic properties through a strong translation-rotation coupling. Then, the acoustic phonons have been studied over five decades in energy and a complete set of elastic constants has been determined. The final section gives some perspectives.

At high temperature, experimental crystal structures of HOP do not reveal strictly ordered and symmetric phases as a consequence of molecular symmetry of the organic cations that do not fit the site symmetry of the lattice. Related dynamical disorder was already analyzed a long time ago by various experimental techniques. ${ }^{15-17}$ Nevertheless, in these studies, their reference high temperature phase can be taken as being the $P m-3 m$ cubic phase, for example, that of $\mathrm{CsPbX}_{3}(Z=1)$. Figure 1a represents the real space $3 \mathrm{D}$ view of the $P m-3 m$ crystal structure of metal-halide $\mathrm{AMX}_{3}$ perovskites, where $\mathrm{A}$ can be an inorganic or an organic cation, $\mathrm{M}$ a metal, and $\mathrm{X}$ a halogen. In 3D HOP, the positions of individual atoms of the organic cation, occupying the same special Wyckoff position at the center of the cubic cell, are in fact dynamically averaged. The $P m-3 m$ space group severely restricts the symmetries and multiplicities of the species located at $(0,0,0)(M, 1 a)$, $\left(\frac{1}{2}, \frac{1}{2}, \frac{1}{2}\right)(\mathrm{A}, 1 \mathrm{~b})$ and $\left(\frac{1}{2}, 0,0\right)(\mathrm{X}, 3 \mathrm{~d})$ sites (Figure 1a). Figure $1 \mathrm{~b}$ describes the corresponding Brillouin zone $(\mathrm{BZ})$ of the primitive cubic Bravais lattice.

Dispersions of the acoustic phonons have been measured using coherent inelastic neutron scattering (INS) and a triple

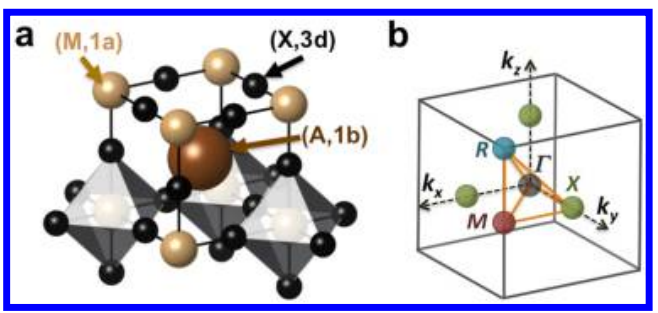

Figure 1. (a) Real space $3 \mathrm{D}$ view of the $P m-3 m$ reference cubic crystal structure of metal-halide AIP or HOP of general formulas $\mathrm{AMX}_{3}$ where $\mathrm{A}$ is an inorganic or an organic cation such as $\mathrm{CH}_{3} \mathrm{NH}_{3}{ }^{+}, \mathrm{M}$ a metal, and $\mathrm{X}$ an halogen. In $\mathrm{HOP}$, the $\mathrm{CH}_{3} \mathrm{NH}_{3}{ }^{+}$cation is located at the center of the cube with an averaged position sketched by the brown ball. (b) Reciprocal space 3D view showing the first Brillouin zone (BZ) of the $P m-3 m$ space group. Points of high symmetry in the cubic BZ are indicated by conventional letters: $\Gamma$ denotes the origin of the BZ; $X$ is the center of a square face at the BZ boundary; $M$ $\left(\frac{1}{2}, \frac{1}{2}, 0\right)$ is a center of a cube edge and $R\left(\frac{1}{2}, \frac{1}{2}, \frac{1}{2}\right)$ corresponds to a vertex of the cube.

axis spectrometer around the (200) Bragg reflection at RT and down to $245 \mathrm{~K}$. Clear acoustic phonon modes are observed on the INS spectra as shown on Figures $2 a$ and S1. Longitudinal acoustic (LA) and transverse acoustic (TA) modes are measured at different reciprocal space positions $\mathbf{Q}=(200)+$ $\mathbf{q}$ in HKL units, with $\mathbf{q}$ along [200], for LA, and $\mathbf{q}$ along the perpendicular to [200] passing through $\mathbf{Q}$ for TA ( $\mathbf{q}$ is parallel to $[011])$. By varying the distance $\mathrm{q}$ to this $\Gamma$ point (Bragg reflection), the dispersion curves can be drawn. The experimental spectra are fitted as follows: a quasi-elastic peak (QE) centered at 0 , a damped harmonic oscillator (DHO) corresponding to the probed phonon mode and a flat background are adjusted. The two fitted peaks are convolved with the apparatus resolution function. Then, sound velocities have been derived from linear regressions of the corresponding dispersion curves (Figure $2 \mathrm{~b}$ and Table 1 ). Measurements were also carried out in the vicinity of the (111) and (011) Bragg reflections but did not show any measurable acoustic phonons peaks along both transverse and longitudinal directions. This is probably due to a very strong damping when going away from $\Gamma$. A second measurement of sound velocity has also been carried out, using an ultrasonic technique that probes LA waves along the [100] direction. This second low frequency technique $(7.46 \mathrm{MHz} / 0.031 \mu \mathrm{eV}$ ) gives a value of the longitudinal velocity along this direction, which is very close to the one obtained by INS. This sound velocity is thus probed over five decades in energy (Figure S4 and Table 1).

BS has been used to study the angular dispersion of acoustic phonons in the vicinity of the normal to a (100) flat surface in the [100], [010] base plane (Figure S5). The maximum angle that has been reached inside the crystal is given by the Brewster incidence. Figure 3 shows LA and TA phonon spectra measured at room temperature for normal and Brewster incidences, showing a large change of the Brillouin shift for TA and to a lesser extent for LA phonons. The optical index is calculated from the measurement of the Brewster angle where the reflected beam vanishes if polarized in the reflection plane. This gives an optical index value of $n=2.1 \pm 0.1$, which is consistent with other experimental measurements. ${ }^{43,44}$

The experimental results are reported in Figure S5 with fitted lines and corresponding elastic constants are given in Table 1 (see technical details in the SI). 

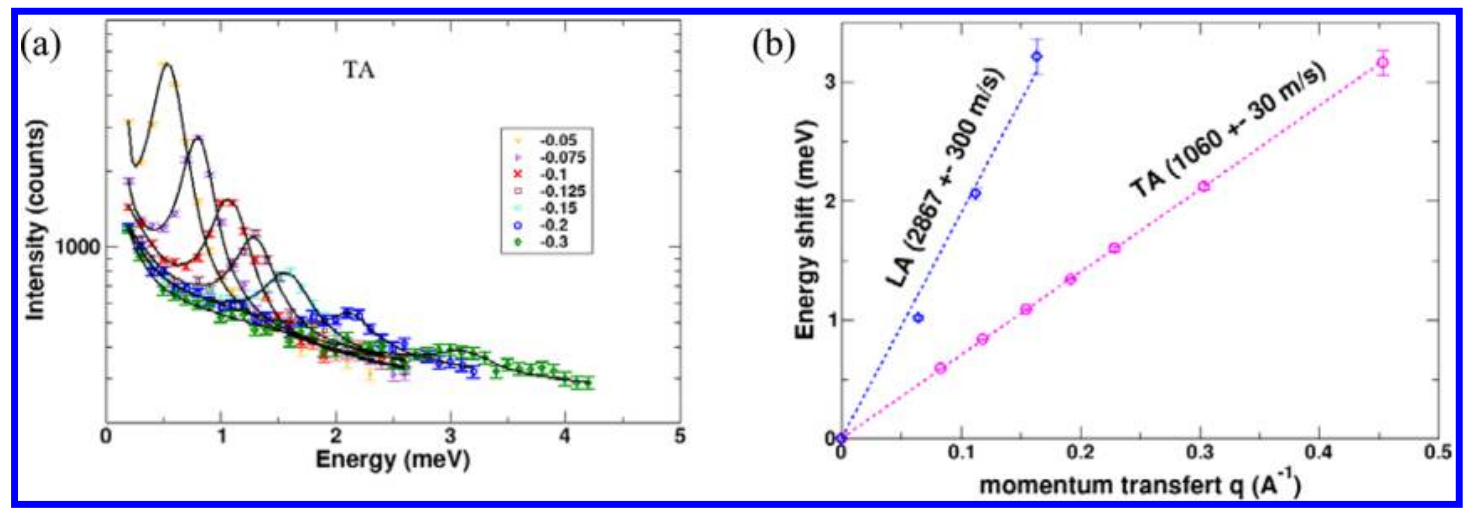

Figure 2. (a) Low energy transverse acoustic (TA) phonon spectra measured by inelastic neutron scattering in the cubic phase of $\mathrm{CH}_{3} \mathrm{NH}_{3} \mathrm{PbBr}_{3}(T$ $=280 \mathrm{~K})$ for different $\mathbf{Q}$ positions going away from the (200) Bragg peak, along the [011] direction (i.e., toward the $M$ point of the $\mathrm{BZ}: \mathbf{Q}=(2 \mathrm{k} k)$, for various $k$ values indicated in the legend) showing a TA mode from low energy to higher energy. (b) Dispersion curves of the TA (Figure 2a) and LA (Figure S1) phonons close to the (200) Bragg peak.

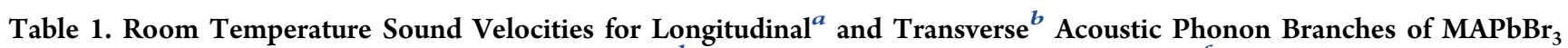
Measured by Brillouin ${ }^{c}$, Coherent Inelastic Neutron ${ }^{d}$, and Ultrasound ${ }^{e}$ Scattering Techniques $^{f}$

$\begin{array}{lccccccccccc} & V_{\mathrm{LA}}\left(\mathrm{m} \cdot \mathrm{s}^{-1}\right) & V_{\mathrm{TA1}}\left(\mathrm{m} \cdot \mathrm{s}^{-1}\right) & V_{\mathrm{TA2}}\left(\mathrm{m} \cdot \mathrm{s}^{-1}\right) & \begin{array}{c}C_{11} \\ (\mathrm{GPa})\end{array} & \begin{array}{c}C_{44} \\ (\mathrm{GPa})\end{array} & \begin{array}{c}C_{12} \\ (\mathrm{GPa})\end{array} & \begin{array}{c}K \\ (\mathrm{GPa})\end{array} & \begin{array}{c}E(100) \\ (\mathrm{GPa})\end{array} & \begin{array}{c}E(111) \\ (\mathrm{GPa})\end{array} & \begin{array}{c}G(100) \\ (\mathrm{GPa})\end{array} & \begin{array}{c}G(111) \\ (\mathrm{GPa})\end{array} \\ \text { BS } & 3075 \pm 150 & 1010 \pm 50 & 1788 \pm 50 & 35.9 & 3.9 & 11.6 & 19.7 & 30.2 & 11 & 3.9 & 6 \\ \text { INS } & 2870 \pm 300 & 1060 \pm 30 & & 31.3 & 4.3 & & & & & & \\ \text { US } & 2900 \pm 200 & & & 32 & & & & & \end{array}$

${ }^{a} V_{\mathrm{LA}}$ (Figure S1). ${ }^{b} V_{\mathrm{TA1}}$ (Figure 2a) and $V_{\mathrm{TA} 2} \cdot{ }^{c} \mathrm{BS} .{ }^{d} \mathrm{INS} .{ }^{e} \mathrm{US} .{ }^{f_{\text {The }}}$ experimental values of the $C_{11}, C_{12}$, and $C_{44}$ elastic constants for MAPbBr 3 in the $P m-3 m$ cubic phase are reported. Sound velocities and elastic constants are respectively given in $\mathrm{m} \cdot \mathrm{s}^{-1}$ and GPa. $K, E$, and $G$ correspond to the bulk, Young, and shear elastic moduli, respectively. The largest relative error is obtained for the $C_{12}$ value, which depends on the measurements of both $V_{\mathrm{LA}}$ and $V_{\mathrm{TA} 2}$ (see SI). For instance, considering $C_{11}=32 \mathrm{GPa}$ (INS) instead of 35.9 (BS) leads to $C_{12}=7.7 \mathrm{GPa}$.

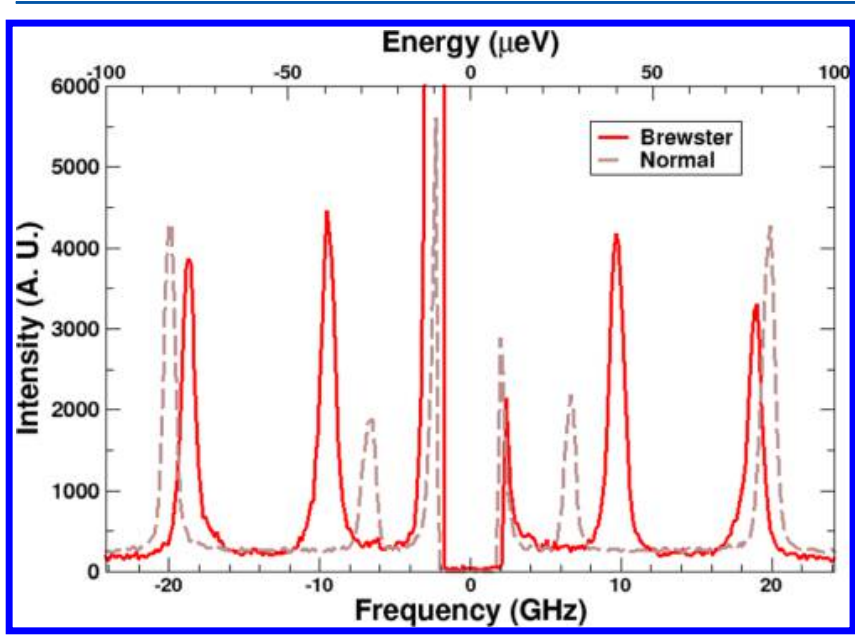

Figure 3. Room temperature Brillouin scattering spectra for normal (gray dashed line) and Brewster angle (red full line) incidences as a function of both frequency $(\mathrm{GHz})$ and energy shift $(\mu \mathrm{eV})$. The Brillouin lines located at about $\pm 80 \mu \mathrm{eV}$ correspond to the longitudinal acoustic (LA) phonon, whereas the lines in the [25-40 $\mu \mathrm{eV}]$ energy range are related to transverse acoustic (TA) phonon.

These values reveal anisotropic crystal properties that are comparable but significantly lower than predicted values $\left(C_{11, \mathrm{DFT}}=47.2, C_{44, \mathrm{DFT}}=8.1\right.$, and $\left.C_{12, \mathrm{DFT}}=10.2 \mathrm{GPa}\right),{ }^{45}$ but in good agreement with recent ultrasonic measurements. ${ }^{30}$ However, DFT predictions are based on a pseudocubic cell with a fixed orientation of the MA cation. Thus, the $P m-3 m$ lattice symmetry is automatically broken, leading to a polar space group. While the elastic stability of the computed structure has already been checked, nothing is reported concerning the stability of the structure with respect to the lattice distortions related to the optical modes. Indeed, these modes are expected to induce the low temperature structural phase transitions. However, the DFT computation, which does not contain entropy contribution, does not account for the stochastic relaxational rotation of the MA cation which leads to the high temperature softening of the elastic properties via the coupling of acoustic phonons to pseudospin elastic quadrupoles (vide infra). ${ }^{36}$ The Zener parameter $A=2 C_{44} /\left(C_{11}-C_{12}\right)$ characterizes the deviation from elastic isotropy $(A=1)$ in a cubic crystal. The experimental value at RT for $\mathrm{MAPbBr}_{3}, A=$ 0.32 , is small and very different from those reported for conventional oxide perovskites, about 1.1, 1.6, and 1.3 for $\mathrm{SrTiO}_{3}, \mathrm{BaTiO}_{3}$, and $\mathrm{PbTiO}_{3}$, respectively. ${ }^{46}$

Mechanical engineers often describe elastic properties by the Young modulus $E$, the Poisson ratio $\nu$ and the shear elastic modulus G. Since cubic structures are not elastically isotropic, these elastic properties depend on strain orientation. An experimental study of mechanical properties based on nanoindentation has been performed on $\mathrm{CH}_{3} \mathrm{NH}_{3} \mathrm{PbI}_{3}$, $\mathrm{CH}_{3} \mathrm{NH}_{3} \mathrm{PbBr}_{3}$, and $\mathrm{CsPbBr}{ }_{3}{ }^{47}$ This study reports a Young modulus along the $[100]$ direction of $E_{[100]}=19.6 \mathrm{GPa}$, whereas the calculated value from the elastic constants (Table $1)$ is $E_{[100]}=C_{11}-2 C_{12}^{2} /\left(C_{11}+C_{12}\right)=30.2 \mathrm{GPa}$. However, the other extreme Young modulus value along [111] is $E_{[111]}=$ $3 C_{44}\left(C_{11}+2 C_{12}\right) /\left(C_{11}+2 C_{12}+C_{44}\right)=11 \mathrm{GPa}($ Table 1$) \cdot \mathrm{A}$ first hypothesis for the discrepancy between both measurements of $E_{[100]}$ may be related to the fact that the nanoindentation technique may lead to an average over Young modulus values close to the [100] direction. A second hypothesis relies on the fact that nanoindentation can be considered as a quasistatic measurement, whereas BS probes 
the elastic properties in the $5-25 \mathrm{GHz}$ frequency range, that is, in the $20-100 \mu \mathrm{eV}$ range (Figure 3). Therefore, the difference between the two experimental $E_{[100]}$ values is likely to result from a frequency dependent softening related to translationrotation coupling (vide infra). This is usually referred to as the difference between the isothermal (low-frequency) and adiabatic (high-frequency) behaviors. On the contrary, the bulk modulus $K=\left(C_{11}+2 C_{12}\right) / 3=19.7 \mathrm{GPa}$ (Table 1$)$ agrees well for both methods yielding a value of about $16 \mathrm{GPa}$ by nanoindentation. This is also consistent with US measurements yielding the same LA velocity as BS at much lower energy $(0.031 \mu \mathrm{eV})$. The difference between the isothermal and adiabatic values of the Young moduli can thus be ascribed mostly to the isothermal and adiabatic $C_{44}$ elastic constants, which does not appear in the expression of the bulk modulus and the LA velocity along the [100] direction (see Supporting Information). A difference between the nanoindentation and the present Brillouin scattering studies exists for the shear modulus, which equals $C_{44} \approx 4 \mathrm{GPa}$ for a $(x-z)$ shear and $\left(C_{11}\right.$ $\left.-C_{12}\right) / 2 \approx 12 \mathrm{GPa}$ for a $(x-y)$ shear according to our measurements, whereas an intermediate shear modulus value of 7.6 GPa was deduced from the indentation experiment. ${ }^{47}$ However, the comparison is less relevant as the nanoindentation shear modulus was deduced by combining, within the isotropic approximation, the experimental Young modulus $(19.6 \mathrm{GPa})$ and a DFT computation of the Poisson's ratio (0.29).

The softening of the elastic properties induced by the translation-rotation coupling may be associated with specific directions of the reciprocal space, as indicated by the INS measurements, which show a clear difference between the LA and TA measurements close to the (200) and (111), (011) Bragg reflections. Finally, let us mention that the formation of small polarons, likely responsible for part of the efficiency drop in photovoltaic devices under illumination, ${ }^{7}$ is expected to be enhanced by the additional softening of the elastic constants through translation-rotation coupling. ${ }^{36,14}$

INS and BS measurements were also carried out below RT to investigate the influence of the cubic $\mathrm{Pm}-3 m$ to tetragonal I4/ $m \mathrm{~cm}$ phase transition of $\mathrm{MAPbBr}_{3}$ on the low energy structural excitations. Our analysis strongly relies on the use of group theory. ${ }^{24,27}$ As indicated in Table 2, the order parameter of the

Table 2. Antiferrodistortive $I 4 / \mathrm{mcm}$ and Pnma Space Group Changes Associated to Nonpolar Order Parameters in $\mathrm{MAPbX}_{3}$ Compounds $^{a}$

\begin{tabular}{|c|c|c|c|c|c|c|}
\hline \multirow[b]{2}{*}{ order parameters } & \multicolumn{3}{|c|}{$M_{3}^{+}$} & \multicolumn{3}{|c|}{$\mathrm{R}_{4}^{+}$} \\
\hline & $\mathbf{q}_{1}$ & $\mathbf{q}_{2}$ & $\mathbf{q}_{3}$ & $\mathbf{q}_{4}$ & $\mathbf{q}_{5}$ & $\mathbf{q}_{6}$ \\
\hline $\mathrm{I} 4 / \mathrm{mcm}$ & 0 & 0 & 0 & $\mathrm{a}$ & 0 & 0 \\
\hline Pnma & 0 & A & 0 & $\mathrm{~b}$ & 0 & $\mathrm{~b}$ \\
\hline $\mathrm{P} 4 / \mathrm{mbm}$ & $\mathrm{A}$ & 0 & 0 & 0 & 0 & 0 \\
\hline
\end{tabular}

${ }^{a} M_{3}{ }^{+}$and $R_{4}{ }^{+}$correspond to in-phase and anti-phase octahedra tilts, respectively. The antiferrodistortive $\mathrm{P} 4 / \mathrm{mbm}$ space group change is observed in the $\mathrm{CsPbCl}_{3}$ perovskite.

phase transition has the $\mathrm{R}_{4}^{+}$symmetry and may correspond to a soft (displacive) phonon with a pure antiphase rotations of $\mathrm{PbBr}_{6}$ octahedra involving only motions of $\mathrm{Br}$ atoms (Table 3). However, previous experimental and theoretical investigations predict an order-disorder character for the structural phase transition. Indeed, the stochastic reorientational motion of the MA cations also span the $R_{4}{ }^{+}$symmetry, yielding an alternative
Table 3. IR decompositions for the phonons in $\mathrm{MAPbX}_{3}$ compounds at the $\Gamma, R$, and $M$ points of the $P m-3 m$ Brillouin zone $(\mathrm{BZ})^{a}$

\begin{tabular}{|c|c|c|c|}
\hline & $\Gamma$ & $R$ & $M$ \\
\hline $\mathrm{Pb}$ atom site $1 \mathrm{a}$ & $\Gamma_{4}^{-}$ & $R_{4}^{-}$ & $M_{3}^{-}+M_{5}^{-}$ \\
\hline $\begin{array}{l}\mathrm{CH}_{3} \mathrm{NH}_{3}^{+} \text {center of } \\
\text { mass site } 1 \mathrm{~b}\end{array}$ & $\Gamma_{4}^{-}$ & $R_{5}^{+}$ & $M_{2}^{-}+M_{5}^{-}$ \\
\hline $\begin{array}{c}\mathrm{X}=\mathrm{I}, \mathrm{Br}, \mathrm{Cl} \text { atoms } \\
\text { site } 3 \mathrm{~d}\end{array}$ & ${ }^{2 \Gamma_{4}{ }^{-}{ }_{5}^{-}}+$ & $\begin{array}{l}R_{1}^{+}+R_{3}^{+}+ \\
R_{4}^{+}+R_{5}^{+}\end{array}$ & $\begin{array}{c}M_{1}^{+}+M_{2}^{+}+M_{3}^{+}+M_{4}^{+}+ \\
M_{5}^{+}+M_{3}^{-}+M_{5}^{-}\end{array}$ \\
\hline
\end{tabular}

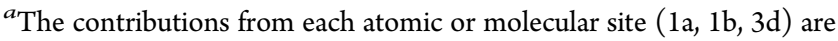
indicated. The symmetric (+) and antisymmetric (-) IR correspond to non-polar and polar phonon modes, respectively.

pseudospin order parameter. ${ }^{16,36,39}$ More, a linear-quadratic coupling between the strain tensor components and the $R_{4}^{+}$ symmetry around the cubic $P m-3 m$ to tetragonal $I 4 / \mathrm{mcm}$ phase transition is predicted from a symmetry analysis. ${ }^{36}$ At lower temperature, $\mathrm{MAPbBr}_{3}$ exhibits a first order and reconstructive structural phase transition from the tetragonal $14 / \mathrm{mcm}$ phase to a Pnma orthorhombic phase (see Swainson et al. for instance). ${ }^{31}$ An intermediate phase between $148.8 \mathrm{~K}$ and 154 $\mathrm{K}$ was also reported by Onoda and co-workers, ${ }^{16}$ between the two phases. The structural instability, which leads to the low temperature Pnma phase, is supposed to be related to the simultaneous condensation of order parameters with $\mathrm{M}_{3}{ }^{+}$and $R_{4}{ }^{+}$symmetries (Table 2, Figure 1). These two IR (irreducible representation) may correspond to the simultaneous softening of phonons with pure antiphase and in-phase rotations of $\mathrm{PbBr}_{6}$ octahedra respectively, or also pseudospin critical fluctuations (Table 3). The $\mathrm{M}_{3}{ }^{+}$order parameter may thus be associated with an order-disorder character of the transition or a soft phonon like in $\mathrm{CsPbl}_{3}$. ${ }^{37}$

Coherent INS experiments were thus performed on optical phonons close to the $R$ (Figure 4) and $M$ (Figure S6) points of the BZ. These experiments did not reveal any measurable change of the optical phonons as a function of the temperature in the cubic and tetragonal phases down to $180 \mathrm{~K}$. As shown on Figure 4 , the spectrum measured at $\left(\frac{3}{2}, \frac{1}{2}, \frac{1}{2}\right)$ exhibits peaky features in the $[6-17 \mathrm{meV}]$ range, consistent with our available Raman scattering data (vide infra) in the low energy range (a similar measurement was performed at the $\left(\frac{3}{2}, \frac{3}{2}, \frac{3}{2}\right)$ and $\left(\frac{1}{2}, \frac{3}{2}, \frac{3}{2}\right)$ positions). Three optical phonons in this energy range are fitted by damped harmonic oscillator (DHO) functions and a background (BG) is added. Fitting parameters are given in the SI. The comparison between the INS intensities at $\left(\frac{3}{2}, \frac{1}{2}, \frac{1}{2}\right)$ and $\left(\frac{1}{2}, \frac{3}{2}, \frac{3}{2}\right)$ shows an enhancement of the INS structure factors between 0 and $10 \mathrm{meV}$ (Figure 4 inset). In order to understand the origin of this low energy feature, it is interesting to recall that a low energy phonon mode $(5 \mathrm{meV})$ was reported by INS in the orthorhombic phase by Swainson et al. ${ }^{33}$ This phonon line corresponds to rotations of the $\mathrm{PbBr}_{6}$ octahedra and undergoes a progressive softening when increasing the temperature toward the orthorhombic to tetragonal transition at $150 \mathrm{~K}$. However, the sharp, harmonic modes in the orthorhombic phase (Pnma) are replaced in the tetragonal phase $(I 4 / \mathrm{mcm})$ by quasistatic fluctuations extending up to high energies at $200 \mathrm{~K}$. A soft phonon line is usually also expected in the high temperature phase for a continuous phase transition. However, the orthorhombic to tetragonal phase transition in $\mathrm{MAPbX}_{3}$ compounds does not fall into this 


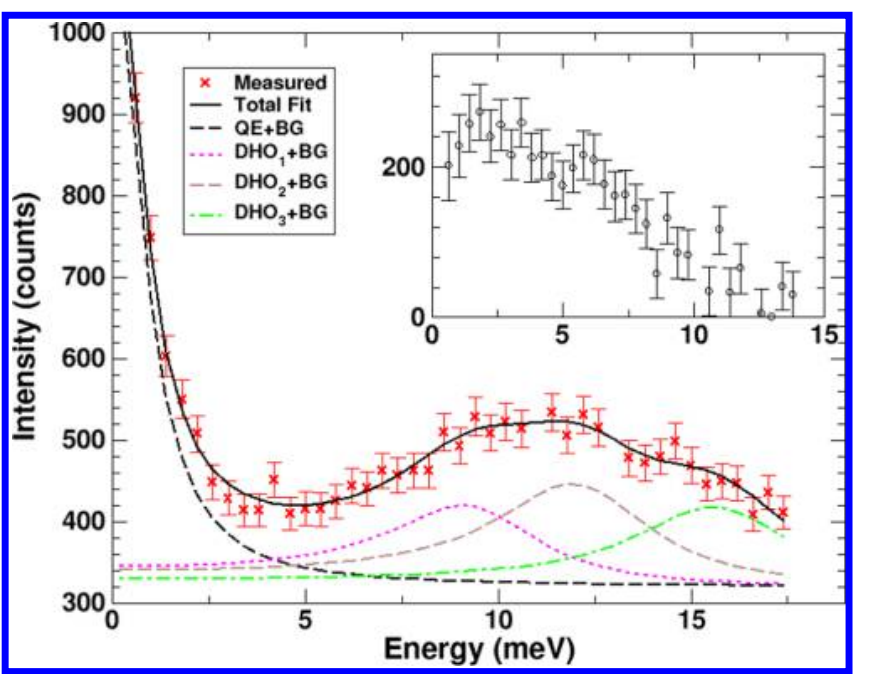

Figure 4. Low energy optical phonon spectra measured by inelastic neutron scattering in the cubic phase of $\mathrm{CH}_{3} \mathrm{NH}_{3} \mathrm{PbBr}_{3}(T=280 \mathrm{~K})$ at various $R$ points of the $\mathrm{BZ}$ reveal two different features: the spectrum measured at the $\left(\frac{3}{2}, \frac{1}{2}, \frac{1}{2}\right)$ point exhibits a quasi-elastic (QE) contribution and three optical phonons (main plot). Optical phonons are fitted by damped harmonic oscillator (DHO) functions and a background (BG) is added. Fitting parameters are given in the SI. The inset shows difference between spectra measured at $\left(\frac{1}{2}, \frac{3}{2}, \frac{3}{2}\right)$ and $\left(\frac{3}{2}, \frac{1}{2}, \frac{1}{2}\right)$, highlighting the enhancement of the $\left(\frac{1}{2}, \frac{3}{2}, \frac{3}{2}\right)$ intensity at low energy. A third measurement carried out at $\left(\frac{3}{2}, \frac{3}{2}, \frac{3}{2}\right)$ did not reveal any enhancement.

category, because it is reconstructive, without group-subgroup relation between the $I 4 / \mathrm{mcm}$ and Pnma space groups. The orthorhombic to tetragonal transition at $150 \mathrm{~K}$ in $\mathrm{MAPbBr}_{3}$ represents thus a case where a displacive $\mathrm{PbBr}_{6}$ octahedra dynamics is replace by an order-disorder MA dynamics, at high temperature. ${ }^{33}$ Indeed, our observations around the tetragonal to cubic phase transition do not show any indications of phonon mode softening. Thus, it is consistent with a purely order-disorder character of the tetragonal to cubic phase transition. More, previous experimental and theoretical determinations of neutron scattering structure factors for $\mathrm{R}_{4}^{+}$ $\mathrm{PbCl}_{6}$ octahedra soft phonons in $\mathrm{CsPbCl}_{3},{ }^{37}$ show similar values at the $\left(\frac{3}{2}, \frac{1}{2}, \frac{1}{2}\right)$ and $\left(\frac{1}{2}, \frac{3}{2}, \frac{3}{2}\right)$ positions contrary to the present results for $\mathrm{MAPbBr}_{3}$ (Figure 4). The low energy feature measured at the $\left(\frac{1}{2}, \frac{3}{2}, \frac{3}{2}\right)$ position (Figure 4 inset) thus probably corresponds to the additional critical quasistatic fluctuations reported by Swainson et al., ${ }^{33}$ between 0 and 10 $\mathrm{meV}$ in the tetragonal phase. No precise indication about the localization in reciprocal space was given in this reference, but the associate $Q$ vector interval corresponds roughly to the one of the present study $Q<5 \AA^{-1}$. Low energy optical phonon spectra measured by INS in the cubic phase of $\mathrm{CH}_{3} \mathrm{NH}_{3} \mathrm{PbBr}_{3}$ $(T=245 \mathrm{~K})$ at the $M$ point (Figure $\left.S 6\left(0, \frac{3}{2}, \frac{3}{2}\right)\right)$ also show a broad quasi-elastic contribution between 0 and $10 \mathrm{meV}$, whereas $M_{3}^{+} \mathrm{PbX}_{6}$ soft phonons should exhibit a structure factor equal to zero. ${ }^{37}$ This observation is consistent with the prediction of the simultaneous condensations of $\mathrm{M}_{3}^{+}$and $\mathrm{R}_{4}^{+}$ order parameters in the orthorhombic Pnma phase, ${ }^{36}$ both having an order-disorder pretransitional dynamics in the high temperature phases of $\mathrm{MAPbX}_{3}$ compounds contrary to $\mathrm{CsPbCl}_{3}$.

Low energy structural excitations in $\mathrm{MAPbBr}_{3}$ single crystals were also explored at RT using Raman scattering. At first sight, classical symmetry-based selection rules for Raman scattering in Pm-3m perovskite crystals lead to the prediction of totally forbidden optical activity for the polar optical lattice phonons at $\Gamma$ point when the $\mathrm{MA}^{+}$cation is replaced by its center of mass (Table 3). Raman activity is thus only expected for high frequency internal modes of the $\mathrm{MA}^{+}$cation. However, it is known that Raman scattering signatures may be obtained at very low frequency in plastic crystals, due to symmetry allowed contributions associated with stochastic molecular relaxational motions. Moreover, we cannot rule out the influence of the large anharmonicity of the iodide motions on the breaking of the Raman scattering selection rules. Such signatures recorded experimentally are clearly visible in Figure 5 . The three independent Raman scattering spectra $\mathrm{A}_{1 \mathrm{~g}}\left(\Gamma_{1}^{+}\right), \mathrm{E}_{\mathrm{g}}\left(\Gamma_{3}^{+}\right)$, and $\mathrm{T}_{2 \mathrm{~g}}\left(\Gamma_{5}{ }^{+}\right)$were obtained at RT by measuring parallel (VV) and crossed $(\mathrm{VH})$ polarized spectra in two different scattering geometries. The symmetry analysis (vide infra) shows that it is possible to compare and calibrate the sum of both $I_{\mathrm{VV}}$ and $I_{\mathrm{VH}}$ spectra for the two scattering geometries (Figure 5-a). This enables to determine accurately the three independent spectra
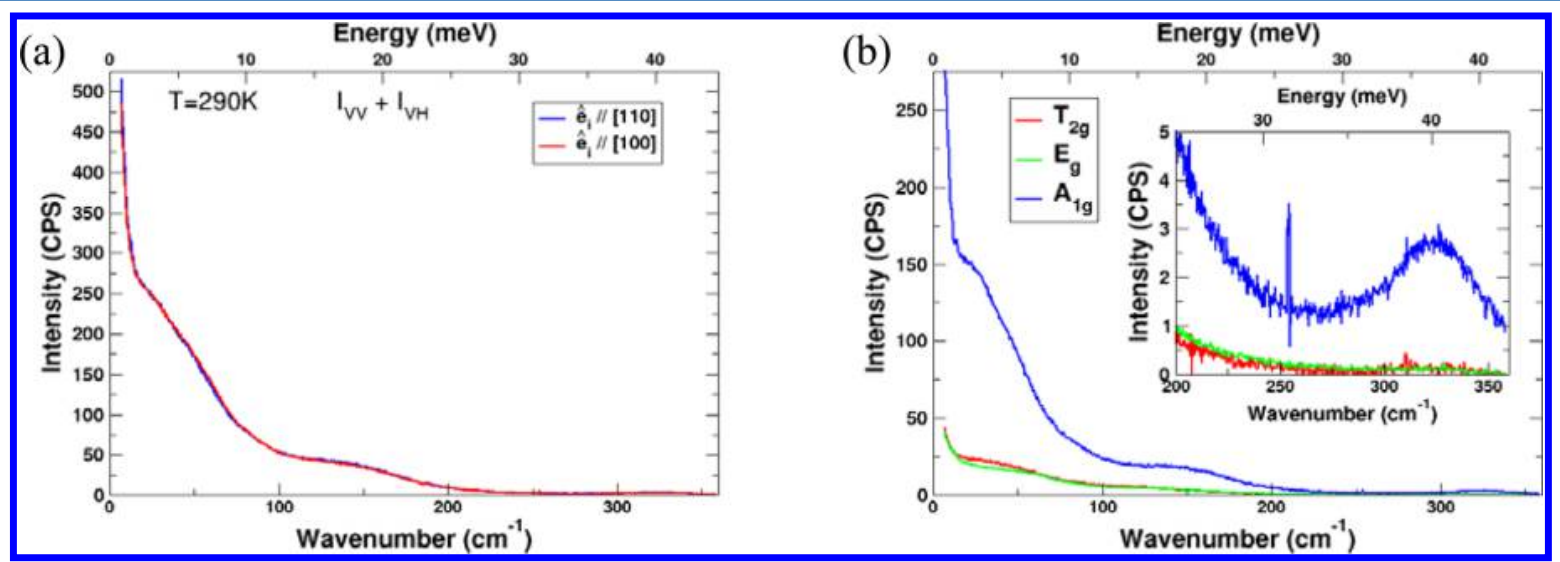

Figure 5. Low energy optical phonon spectra measured by Raman scattering in the cubic phase of $\mathrm{CH}_{3} \mathrm{NH}_{3} \mathrm{PbBr}_{3}$ at room temperature. (a) Total contribution of parallel $\left(I_{\mathrm{VV}}\right)$ and crossed $\left(I_{\mathrm{VH}}\right)$ polarized intensities with propagation directions along [001] and incoming polarization along [100] or [110]. (b) Raman spectra of $T_{2 g}, E_{g}$ and $A_{1 g}$ modes. Inset: zoom to highlight an $A_{1 g}$ optical mode near $40 \mathrm{meV}$. 
$\left(A_{1 g}, E_{g}\right.$ and $T_{2 g} ;$ Figure 5-b). The $A_{1 g}$ spectrum largely dominates, while $E_{g}$ and $T_{2 g}$ spectra reveal comparable intensities. All, the three spectra show broad bands and the $A_{1 g}$ spectrum is superimposed on an intense quasi-elastic component. This observation is consistent with previous theoretical modeling of tumbling relaxational motions of cations in $\mathrm{MAPbX}_{3}$, using symmetry-adapted discrete pseudospins or continuous rotators. ${ }^{36,39}$ Indeed, relaxational modes include polar modes, with contributions to the lattice dielectric constants, but also nonpolar modes (elastic multipoles) with $\mathrm{A}_{1 \mathrm{~g}}\left(\Gamma_{1}^{+}\right), \mathrm{E}_{\mathrm{g}}\left(\Gamma_{3}^{+}\right)$, and $\mathrm{T}_{2 \mathrm{~g}}\left(\Gamma_{5}^{+}\right)$symmetries. The inset highlights the high frequency part of the measured spectra showing an $\mathrm{A}_{1 \mathrm{~g}}$ optical mode at about $40 \mathrm{meV}$. This mode may be tentatively assigned to a symmetric internal mode of the $\mathrm{CH}_{3} \mathrm{NH}_{3}{ }^{+}$cation.

Temperature-dependent Brillouin backscattering measurements allow probing the coupling between low energy relaxational modes and phonons at lower energies than Raman scattering (Figure 6). The LA phonon mode exhibits

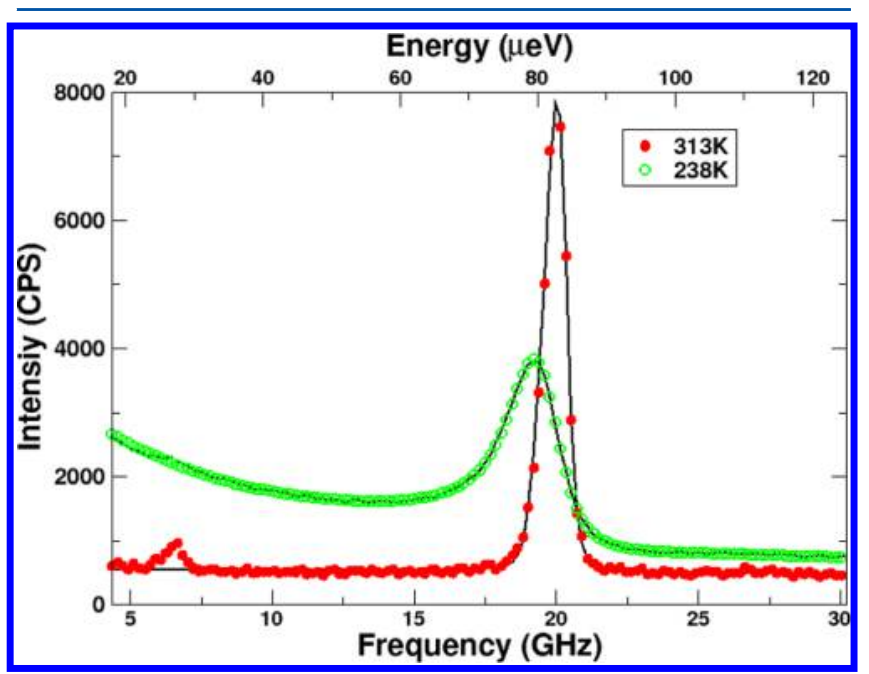

Figure 6. Brillouin scattering spectra of $\mathrm{MAPbBr}_{3}$ for normal incidence above $(T=313 \mathrm{~K})$ and very close to the transition temperature $(238 \mathrm{~K})$. The LA phonon mode exhibits an anomalous increase of its damping as the temperature is lowered. The TA phonon mode is very weak due to restrictive normal incidence conditions when performing the low temperature measurements, as compared to the setup used at RT (Figure 3).

an anomalous increase of its damping as the temperature is lowered. This behavior is interpreted as the direct consequence of the coupling between acoustic modes and low energy relaxational modes. Moreover, the LA phonon mode at $T=238$ $\mathrm{K}$ displays an asymmetric shape which can only be explained by a coupling to a relaxational mode. Spectra have thus been fitted using a DHO peak for the LA acoustic phonon mode and two relaxational components: a large quasi-elastic (LQE) one and a central peak (CP), at very low energy. The widths (HWHM) of these two features correspond to very different characteristic relaxation times. Finally a flat background (B) fixed to the detector thermal noise was added. A good fitting of the data was obtained by assuming that the $\mathrm{CP}$ is coupled to the $\mathrm{DHO}$ peak via two adjustable parameters $\delta$ and $\beta\left(\delta^{2}\right.$ represents the coupling strength and $\beta^{2}$ the $\mathrm{CP} / \mathrm{DHO}$ intensity ratio). Then the intensity is computed from ${ }^{48}$

$$
I(E)=A \cdot b(T) \cdot \chi_{\mathrm{T}}^{\prime \prime}(E)+B
$$

where $\chi_{\mathrm{T}}^{\prime \prime}(E)$ is the imaginary part of the coupled optical susceptibility (see Supporting Information). As shown in Figure S7, a significant lowering of the LA Brillouin shift is observed after correction of the coupling with CP. Moreover, the coupling strength between the LA phonon and the central relaxational mode is highest at the phase transition temperature (Figure S8). Both observations are consistent with a linearquadratic coupling between the strain and the order parameter of the phase transition, which exhibit an order-disorder character.

This picture is further illustrated in Figure 7, where the widths of the CP and the LQE are reported as a function of the

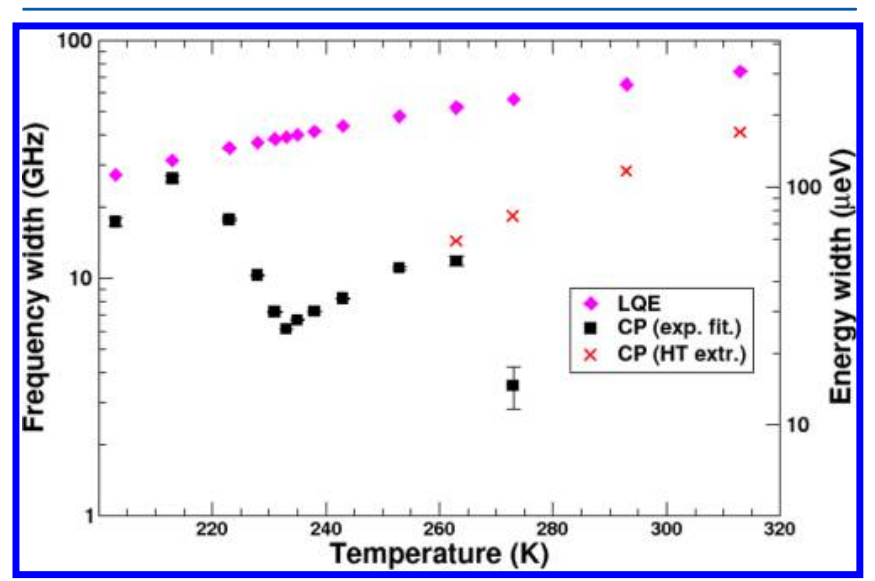

Figure 7. Frequency widths (HWHM) of the LQE and CP quasielastic peaks as a function of the temperature. Low temperature $\mathrm{CP}$ widths above the transition are fitted using an Arrhenius law, allowing extrapolation of high temperature values (red crosses).

temperature. The evolution of the two different widths seems to be in line with the scenario proposed by Chen al for $\mathrm{CH}_{3} \mathrm{NH}_{3} \mathrm{PbI}_{3} .{ }^{40}$ They describe a rotational model of the $\mathrm{CH}_{3} \mathrm{NH}_{3}{ }^{+}$molecule, associated with two relaxational motions, the slowest one being related to the tumbling of the $\mathrm{C}-\mathrm{N}$ axis and the fastest one with the rotation around the same axis. Therefore, the LQE observed in the present BS experiments on $\mathrm{MAPbBr}_{3}$ may be attributed to the fast motion of the hydrogen atoms around the $\mathrm{C}-\mathrm{N}$ axis, whereas the $\mathrm{CP}$ can be tentatively assigned to the tumbling motion of the cations. Then, the characteristic relaxation times can be calculated from $\Gamma=\frac{h}{2 \pi \tau} \approx \frac{658}{\tau}$ when the energy is expressed in $\mu \mathrm{eV}$ and the time in ps. The corresponding relaxation time at $T_{\mathrm{c}}$, deduced from the present study on $\mathrm{MAPbBr}_{3}$, (Figure 7) is about $28 \mathrm{ps}$ (CP). In $\mathrm{MAPbI}_{3}$, the relaxation times reported by the INS study being equal to about $3 \mathrm{ps}$ for the $\mathrm{CP}$ at $T_{\mathrm{c}}(330 \mathrm{~K}) .^{40} \mathrm{In}$ $\mathrm{MAPbBr}_{3}$, the CP obtained by BS exhibits an anomaly around $T_{\mathrm{c}}$ in the $[220-240 \mathrm{~K}]$ temperature range (Figure 7 ). We may notice that the $\mathrm{CP}$ obtained in $\mathrm{MAPbI}_{3}$ by INS is also exhibiting a discontinuity at the cubic to tetragonal phase transition, although a precise comparison with the present work is difficult since the INS study displays very few experimental points in the same temperature range close to $T_{c^{*}}{ }^{40}$ The CP anomalies close to $T_{\mathrm{c}}$ in $\mathrm{MAPbX}_{3}$ compounds should be investigated more precisely in the future using other experimental techniques.

The relaxation times reported for the two compounds using various techniques are gathered in Table 4 . The tumbling of the $\mathrm{C}-\mathrm{N}$ axis is frozen in the orthorhombic Pnma phase of $\mathrm{MAPbI}_{3}$, whereas the motion of the hydrogen atoms around 
Table 4. Relaxation Times Corresponding to CP from Different Sources

\begin{tabular}{lllll} 
material & \multicolumn{1}{c}{ technique } & \multicolumn{1}{c}{$T(\mathrm{~K})$} & $\tau_{\mathrm{CP}}(\mathrm{ps})$ & \multicolumn{1}{c}{ phase } \\
$\mathrm{MAPbI}_{3}$ & $\mathrm{INS}^{41}$ & $\mathrm{RT}$ & 14 & tetra \\
$\mathrm{MAPbI}_{3}$ & $\mathrm{INS}^{40}$ & $\mathrm{RT}$ & 4.7 & tetra \\
$\mathrm{MAPbI}_{3}$ & $\mathrm{INS}^{40}$ & 350 & 2.7 & cubic \\
$\mathrm{MAPbI}_{3}$ & $\mathrm{INS}^{40}$ & $T_{\mathrm{c}}=330$ & 3 & cubic-tetra \\
$\mathrm{MAPbI}_{3}$ & $\mathrm{INS}^{40}$ & $T=230$ & $\sim 25$ & tetra \\
$\mathrm{MAPbI}_{3}$ & millimeter-wave & $\mathrm{RT}$ & 5.4 & tetra \\
$\mathrm{MAPbI}_{3}$ & molecular dynamics & 400 & 10.5 & cubic \\
$\mathrm{MAPbBr}_{3}$ & millimeter-wave & $\mathrm{RT}$ & 2.7 & cubic \\
$\mathrm{MAPbBr}_{3}$ & Brillouin, this work $^{15}$ & $T_{\mathrm{c}}=230$ & 28 & cubic-tetra \\
$\mathrm{MAPbBr}_{3}$ & Brillouin, this work & $\mathrm{RT}$ & 5 & cubic \\
$\mathrm{MAPbBr}_{3}$ & INS & 165 & 131 & tetra \\
\hline
\end{tabular}

the $\mathrm{C}-\mathrm{N}$ axis is still effective. ${ }^{40}$ A further slowing down of the two relaxational modes may be inferred below $230 \mathrm{~K}$ for $\mathrm{MAPbBr}_{3}$ (Figure 7). Unfortunately, a complete study around the tetragonal to orthorhombic phase transition at $150 \mathrm{~K}$ was not possible due to the presence of multiple strained domains, and thus multiple acoustic phonon lines below $T=200 \mathrm{~K}$.

Through combined neutron, Brillouin and Ultra Sound scattering measurements, we were able to measure for the first time the transverse and longitudinal sound velocities of $\mathrm{CH}_{3} \mathrm{NH}_{3} \mathrm{PbBr}_{3}$ perovskite single crystals allowing RT evaluation of the three cubic elastic constants. It reveals a very low value of the $C_{44}$ shear constant. A further softening is observed by Brillouin scattering close to the cubic to tetragonal phase transition, by a low energy coupling between the LA phonon mode and a CP. This CP is attributed to a relaxation mode of the $\mathrm{CH}_{3} \mathrm{NH}_{3}{ }^{+}$molecule (tumbling around the $\mathrm{C}-\mathrm{N}$ axis), which is consistent with an order-disorder character of the structural phase transition. Broad quasi-elastic excitations and low energy optical phonons were also observed for the first time using INS, confirming the large anharmonicity of the perovskite lattice modes. These optical modes do not exhibit a displacive behavior in the cubic and tetragonal phases, but Raman scattering, INS and Brillouin scattering are compatible with an order-disorder character related to the stochastic relaxational motions of the $\mathrm{MA}^{+}$cations. This disorder leads to a breaking of the Raman scattering selection rules in the high temperature phase, characteristic of a plastic crystal phase.

\section{EXPERIMENTAL METHODS}

Various single crystals of $\mathrm{MAPbBr}_{3}$ have been synthesized, purified and grown according to Saidaminov et al., ${ }^{49}$ with sizes up to $\sim 1 \mathrm{~cm}^{3}$ (see Supporting Information). Concerning the Brillouin scattering experiments, spectra were recorded at 647.1 $\mathrm{nm}$ by using a krypton ion laser and a tandem of Fabry-Perot interferometers where each interferometer is triple-passed giving a contrast larger than $10^{11} .^{50}$ Different free spectral ranges between $10 \mathrm{~cm}^{-1}\left(1 \mathrm{~cm}^{-1}=30 \mathrm{GHz}, 8 \mathrm{~cm}^{-1}=1 \mathrm{meV}\right)$ and $1 \mathrm{~cm}^{-1}$ have been used in order to give a full description of the low-frequency spectra. The advantage of this tandem of interferometers over the single one (see for instance Sander$\operatorname{cock}^{51}$ ) is that it removes the periodicity of the interferogram allowing the observation of broad and narrow components in the same spectrum without any confusion due to order overlapping. The polarization of the incident light was rotated by a half-wave plate and filtered by a Glan prism, whereas the scattered beam was not analyzed.
Coherent INS experiment has been performed using the triple-axis spectrometer 4F1 located on a cold-neutron source at the reactor Orphée (Laboratoire Léon Brillouin, CEASaclay). ${ }^{52}$ The incident and scattered beams were focused by a pyrolytic graphite (002) double-monochromator and analyzer, respectively. The collimation conditions were open. The final neutron wave vector was held fixed at $k_{\mathrm{f}}=1.55 \AA^{-1}$. A beryllium filter cooled with liquid nitrogen was placed after the sample to remove high-order neutrons from the beam. The resolution function was of the order of $0.2 \mathrm{meV}$. Here, the sample is protonated, yielding a large background due to the incoherent neutron cross section of hydrogen. The sample was mounted on a close-cycle refrigerator installed on the spectrometer sample table, ensuring temperature stability less than $0.1 \mathrm{~K}$. The sample was mounted in a scattering plane such that reciprocal directions $(1,0,0)$ and $(0,1,1)$ were within the horizontal plane. We quote the wave-vector $\mathbf{Q}=(H, K, L)$ in units of cubic lattice vector $a^{*}=2 \pi / a$, where $a$ is the lattice parameter $(a=5.91 \AA$ and $a^{*} \approx 1.06 \AA^{-1}$ at $\mathrm{RT}$ ). Bragg reflections of the RT cubic phase $\mathbf{Q}=(H, K, K)$ are then accessible. This has been chosen in order to reach the superlattice reflections appearing in both the tetragonal and orthorhombic low temperature phases, which are occurring at the $M\left(\frac{1}{2}, \frac{1}{2}, 0\right)$ and $R\left(\frac{1}{2}, \frac{1}{2}, \frac{1}{2}\right)$ or equivalents points.

Ultrasonic time-domain reflectometry measurements have been carried out at RT using a longitudinal probe at $7.46 \mathrm{MHz}$. A smaller single crystal $\left(\sim 0.2 \mathrm{~cm}^{3}\right)$ was carefully chosen in order to yield a (001) flat surface with a crystal thickness equal to $3 \mathrm{~mm}$. A thin $(<0.1 \mathrm{~mm})$ gel layer was used between the probe and the crystal in order to ensure a maximum transmission and reflection of the ultrasonic pulse.

The polarized Raman scattering spectra covering the frequency range from 7 to $358 \mathrm{~cm}^{-1}$ with a resolution of about $2 \mathrm{~cm}^{-1}$ (full width at half-maximum) were obtained on a triple monochromator Jobin Yvon T64000 spectrometer equipped with a liquid-nitrogen-cooled silicon CCD detector. A linearly polarized diode laser emitting at $660 \mathrm{~nm}$ was used to excite the sample and the scattered light was collected through a $50 \times$ objective in the backscattering configuration. The laser power impinging the sample with a spot diameter of $3 \mu \mathrm{m}$ was kept below $1 \mathrm{~mW}$ to avoid heating effects. An analyzer was used to select the outgoing polarization, followed by a rotating halfwave plate to maintain a vertical polarization at the entrance of the spectrometer. The polarization-dependent transmission of the collecting optics was calibrated using the known depolarization ratio of the $T_{2 g}$ Raman mode measured in backscattering on a [111] silicon plate: $I_{\mathrm{VH}} / I_{\mathrm{VV}}=2 / 3$. The two $\mathrm{MAPbBr}_{3}$ samples used for the Raman scattering experiment were single-crystal plates $\left(\sim 4 \times 4 \times 1 \mathrm{~mm}^{3}\right)$ with the surface oriented perpendicularly to the [001]-type cubic direction. Setting the polarization of the incoming beam along [100] and measuring parallel and crossed polarized intensities of the outgoing light leads to the first two spectra $S_{1}$ and $S_{2}$

$$
\begin{aligned}
& \mathrm{S}_{1}=\mathrm{A}_{1 \mathrm{~g}}+4 \mathrm{E}_{\mathrm{g}} Z(X, X) \bar{Z} \\
& \mathrm{~S}_{2}=\mathrm{T}_{2 \mathrm{~g}} Z(X, Y) \bar{Z}
\end{aligned}
$$

A rotation of the incoming beam polarization along [110] gives the other two spectra $S_{3}$ and $S_{4}$

$$
\mathrm{S}_{3}=\mathrm{A}_{1 \mathrm{~g}}+\mathrm{E}_{\mathrm{g}}+\mathrm{T}_{2 \mathrm{~g}} Z(X+Y, X+Y) \bar{Z}
$$




$$
\mathrm{S}_{4}=3 \mathrm{E}_{\mathrm{g}} Z(X+Y, X-Y) \bar{Z}
$$

It is interesting to note that for both sets of geometry sums of parallel and crossed polarized spectra are expected to be identical, that is, $S_{1}+S_{2}=S_{3}+S_{4}=A_{1 g}+4 E_{g}+T_{2 g}$. We used this property to obtain the intensity scale factor between the two scattering geometries.

\section{ASSOCIATED CONTENT}

\section{S Supporting Information}

The Supporting Information is available free of charge on the ACS Publications website at DOI: 10.1021/acs.jpclett.6b01709.

Details about sample growth; acoustic dispersion curves measured by neutron scattering; ultrasonic measurements; Brillouin scattering; optical phonons measured at the $M$ and $R$ points by neutron scattering; low temperature Brillouin scattering experiments. (PDF)

\section{AUTHOR INFORMATION}

\section{Corresponding Authors}

*E-mail: Antoine.Letoublon@insa-rennes.fr. Tel.: +33 (0)2 23 2382 95. Fax: +33 (0)2 23238618.

*E-mail: Jacky.Even@insa-rennes.fr. Tel.: +33 (0)2 232382 95. Fax: +33 (0)2 23238618 .

Notes

The authors declare no competing financial interest.

\section{ACKNOWLEDGMENTS}

The work is supported by Agence Nationale pour la Recherche (SUPERSANSPLOMB project no. ANR-15-CE05-0023-02). This project has received funding from the European Union's Horizon 2020 research and innovation program under the grant agreement no. 687008. The information and views set out in this publication are those of the authors and do not necessarily reflect the official opinion of the European Union. Neither the European Union institutions and bodies nor any person acting on their behalf may be held responsible for the use which may be made of the information contained therein. A.L. thanks T. Roisnel, CDIFX, and V. Demange, CSM, at Institut des Sciences Chimiques de Rennes, for X-ray diffraction characterization of the samples. T.M. acknowledges financial support from University of Montpellier "Soutien à la recherche" grant.

\section{REFERENCES}

(1) Kojima, A.; Teshima, K.; Shirai, Y.; Miyasaka, T. Organometal Halide Perovskites as Visible-Light Sensitizers for Photovoltaic Cells. $L$. Am. Chem. Soc. 2009, 131, 6050-6051.

(2) Lee, M. M.; Teuscher, J.; Miyasaka, T.; Murakami, T. N.; Snaith, H. J. Efficient Hybrid Solar Cells Based on Meso-Superstructured Organometal Halide Perovskites. Science 2012, 338, 643-647.

(3) Kim, H.-S.; Lee, C.-R.; Im, J.-H.; Lee, K.-B.; Moehl, T.; Marchioro, A.; Moon, S.-J.; Humphry-Baker, R.; Yum, J.-H.; Moser, J. E.; et al. Lead Iodide Perovskite Sensitized All-Solid-State Submicron Thin Film Mesoscopic Solar Cell with Efficiency Exceeding 9\%. Sci. Rep. 2012, 2, 591.

(4) Liu, M.; Johnston, M. B.; Snaith, H. J. Efficient Planar Heterojunction Perovskite Solar Cells by Vapour Deposition. $\underline{\text { Nature }}$ 2013, 501, 395-398.

(5) Nie, W.; Tsai, H.; Asadpour, R.; Blancon, J.-C.; Neukirch, A. J.; Gupta, G.; Crochet, J. J.; Chhowalla, M.; Tretiak, S.; Alam, M. A.; et al. High-Efficiency Solution-Processed Perovskite Solar Cells with Millimeter-Scale Grains. Science 2015, 347, 522-525.
(6) Even, J.; Pedesseau, L.; Jancu, J.-M.; Katan, C. Importance of Spin-Orbit Coupling in Hybrid Organic/Inorganic Perovskites for Photovoltaic Applications. I. Phvs. Chem. Lett. 2013, 4, 2999-3005.

(7) Nie, W.; Blancon, J.-C.; Neukirch, A. J.; Appavoo, K.; Tsai, H.; Chhowalla, M.; Alam, M. A.; Sfeir, M. Y.; Katan, C.; Even, J.; et al. Light-Activated Photocurrent Degradation and Self-Healing in Perovskite Solar Cells. Nat. Commun. 2016, 7, 11574.

(8) Tsai, H.; Nie, W.; Blancon, J.-C.; Stoumpos, C. C.; Asadpour, R.; Harutyunyan, B.; Neukirch, A. J.; Verduzco, R.; Crochet, J. J.; Tretiak, S.; et al. High-Efficiency Two-Dimensional Ruddlesden-Popper Perovskite Solar Cells. Nature 2016, 536, 312-316.

(9) Bi, D.; Tress, W.; Dar, M. I.; Gao, P.; Luo, J.; Renevier, C.; Schenk, K.; Abate, A.; Giordano, F.; Baena, J.-P. C.; et al. Efficient Luminescent Solar Cells Based on Tailored Mixed-Cation Perovskites. Sci. Adv. 2016, 2, e1501170.

(10) Saliba, M.; Matsui, T.; Seo, J.-Y.; Domanski, K.; Correa-Baena, J.-P.; Nazeeruddin, M. K.; Zakeeruddin, S. M.; Tress, W.; Abate, A.; Hagfeldt, A.; et al. Cesium-Containing Triple Cation Perovskite Solar Cells: Improved Stability, Reproducibility and High Efficiency. Energy Environ. Sci. 2016, 9, 1989-1997.

(11) Tan, Z.-K.; Moghaddam, R. S.; Lai, M. L.; Docampo, P.; Higler, R.; Deschler, F.; Price, M.; Sadhanala, A.; Pazos, L. M.; Credgington, D.; et al. Bright Light-Emitting Diodes Based on Organometal Halide Perovskite. Nat. Nanotechnol. 2014, 9, 687-692.

(12) Even, J.; Pedesseau, L.; Jancu, J.-M.; Katan, C. DFT and K · P Modelling of the Phase Transitions of Lead and Tin Halide Perovskites for Photovoltaic Cells. Phvs. Status Solidi RRL 2014, 8, $31-35$.

(13) Even, J.; Pedesseau, L.; Katan, C. Analysis of Multivalley and Multibandgap Absorption and Enhancement of Free Carriers Related to Exciton Screening in Hybrid Perovskites. I. Phys. Chem. C 2014, $118,11566-11572$.

(14) Neukirch, A. J.; Nie, W.; Blancon, J.-C.; Appavoo, K.; Tsai, H.; Sfeir, M. Y.; Katan, C.; Pedesseau, L.; Even, J.; Crochet, J. J.; et al. Polaron Stabilization by Cooperative Lattice Distortion and Cation Rotations in Hybrid Perovskite Materials. Nano Lett. 2016, 16, 38093816.

(15) Poglitsch, A.; Weber, D. Dynamic Disorder in Methylammoniumtrihalogenoplumbates (II) Observed by Millimeter-wave Spectroscopy. I. Chem. Phvs. 1987, 87, 6373-6378.

(16) Onoda-Yamamuro, N.; Matsuo, T.; Suga, H. Dielectric Study of CH3NH3PbX3 (X = Cl, Br, I). I. Phys. Chem. Solids 1992, 53, 935939.

(17) Wasylishen, R. E.; Knop, O.; Macdonald, J. B. Cation Rotation in Methylammonium Lead Halides. Solid State Commun. 1985, 56, $581-582$.

(18) Weller, M. T.; Weber, O. J.; Henry, P. F.; Di Pumpo, A. M.; Hansen, T. C. Complete Structure and Cation Orientation in the Perovskite Photovoltaic Methylammonium Lead Iodide between 100 and $352 \mathrm{~K}$. Chem. Commun. 2015, 51, 4180-4183.

(19) Lee, J.-H.; Bristowe, N. C.; Bristowe, P. D.; Cheetham, A. K. Role of Hydrogen-Bonding and Its Interplay with Octahedral Tilting in CH3NH3PbI3. Chem. Commun. 2015, 51, 6434-6437.

(20) Bakulin, A. A.; Selig, O.; Bakker, H. J.; Rezus, Y. L. A.; Müller, C.; Glaser, T.; Lovrincic, R.; Sun, Z.; Chen, Z.; Walsh, A.; et al. RealTime Observation of Organic Cation Reorientation in Methylammonium Lead Iodide Perovskites. I. Phvs. Chem. Lett. 2015, 6, 36633669.

(21) Pérez-Osorio, M. A.; Milot, R. L.; Filip, M. R.; Patel, J. B.; Herz, L. M.; Johnston, M. B.; Giustino, F. Vibrational Properties of the Organic-Inorganic Halide Perovskite $\mathrm{CH}_{3} \mathrm{NH}_{3} \mathrm{PbI}_{3}$ from Theory and Experiment: Factor Group Analysis, First-Principles Calculations, and Low-Temperature Infrared Spectra. L. Phvs. Chem. C 2015, 119, 25703-25718.

(22) Yaffe, O.; Guo, Y.; Hull, T.; Stoumpos, C. C.; Tan, L. Z.; Egger, D. A.; Zheng, F.; Szpak, G.; Semonin, O. E.; Beecher, A. N.; et al. The Nature of Dynamic Disorder in Lead Halide Perovskite Crystals. 2016, arXiv:1604.08107. arXiv.org e-Print archive. http://arxiv.org/abs/ 1604.08107 (accessed Sept 5, 2016). 
(23) Brivio, F.; Frost, J. M.; Skelton, J. M.; Jackson, A. J.; Weber, O. J.; Weller, M. T.; Goñi, A. R.; Leguy, A. M. A.; Barnes, P. R. F.; Walsh, A. Lattice Dynamics and Vibrational Spectra of the Orthorhombic, Tetragonal, and Cubic Phases of Methylammonium Lead Iodide. Phys. Rev. B: Condens. Matter Mater. Phys. 2015, 92, 144308.

(24) Sendner, M.; Nayak, P. K.; Egger, D. A.; Beck, S.; Müller, C.; Epding, B.; Kowalsky, W.; Kronik, L.; Snaith, H. J.; Pucci, A. Optical Phonons in Methylammonium Lead Halide Perovskites and Implications for Charge Transport. 2016, ArXiv:1607.08541. arXiv.org e-Print archive. http://arxiv.org/abs/1607.08541 (accessed Sept 5, 2016).

(25) Leguy, A. M. A.; Goñi, A. R.; Frost, J. M.; Skelton, J.; Brivio, F.; Rodríguez-Martínez, X.; Weber, O. J.; Pallipurath, A.; Alonso, M. I.; Campoy-Quiles, M.; et al. Dynamic Disorder, Phonon Lifetimes, and the Assignment of Modes to the Vibrational Spectra of Methylammonium Lead Halide Perovskites. Phvs. Chem. Chem. Phvs. 2016, DOI: $10.1039 / \mathrm{C} 6 \mathrm{CP} 03474 \mathrm{H}$.

(26) Glaser, T.; Müller, C.; Sendner, M.; Krekeler, C.; Semonin, O. E.; Hull, T. D.; Yaffe, O.; Owen, J. S.; Kowalsky, W.; Pucci, A.; et al. Infrared Spectroscopic Study of Vibrational Modes in Methylammonium Lead Halide Perovskites. I. Phvs. Chem. Lett. 2015, 6, 29132918.

(27) Roiland, C.; Trippé-Allard, G.; Jemli, K.; Alonso, B.; Ameline, J.C.; Gautier, R.; Bataille, T.; Le Pollès, L.; Deleporte, E.; Even, J.; et al. Multinuclear NMR as a Tool for Studying Local Order and Dynamics in $\mathrm{CH} 3 \mathrm{NH} 3 \mathrm{PbX} 3(\mathrm{X}=\mathrm{Cl}, \underline{\mathrm{Br}}, \mathrm{I})$ Hybrid Perovskites. Phys. Chem. Chem. Phvs. 2016, DOI: 10.1039/C6CP02947G.

(28) Fang, H.-H.; Raissa, R.; Abdu-Aguye, M.; Adjokatse, S.; Blake, G. R.; Even, J.; Loi, M. A. Photophysics of Organic-Inorganic Hybrid Lead Iodide Perovskite Single Crystals. Adv. Funct. Mater. 2015, 25, 2378-2385.

(29) Even, J.; Paofai, S.; Bourges, P.; Letoublon, A.; Cordier, S.; Durand, O.; Katan, C. Carrier Scattering Processes and Low Energy Phonon Spectroscopy in Hybrid Perovskites Crystals. Proc. SPIE 2016, 9743, 97430M.

(30) Lomonosov, A. M.; Yan, X.; Sheng, C.; Gusev, V. E.; Ni, C.; Shen, Z. Exceptional Elastic Anisotropy of Hybrid Organic-inorganic Perovskite $\mathrm{CH}_{3} \mathrm{NH}_{3} \mathrm{PbBr}_{3}$ Measured by Laser Ultrasonic Technique. Phys. Status Solidi RRL 2016, 10, 606-612.

(31) Swainson, I. P.; Hammond, R. P.; Soullière, C.; Knop, O.; Massa, W. Phase Transitions in the Perovskite Methylammonium Lead Bromide, CH3ND3PbBr3. I. Solid State Chem. 2003, 176, 97-104.

(32) Kawamura, Y.; Mashiyama, H.; Hasebe, K. Structural Study on Cubic-Tetragonal Transition of CH3NH3PbI3. I. Phys. Soc. Ipn 2002, 71, 1694-1697.

(33) Swainson, I. P.; Stock, C.; Parker, S. F.; Van Eijck, L.; Russina, M.; Taylor, J. W. From Soft Harmonic Phonons to Fast Relaxational Dynamics in $\mathrm{CH}_{3} \mathrm{NH}_{3} \mathrm{PbBr}_{3}$. Phvs. Rev. B: Condens. Matter Mater. Phvs. 2015, 92, 100303.

(34) Baikie, T.; Barrow, N. S.; Fang, Y.; Keenan, P. J.; Slater, P. R.; Piltz, R. O.; Gutmann, M.; Mhaisalkar, S. G.; White, T. J. A Combined Single Crystal Neutron/X-Ray Diffraction and Solid-State Nuclear Magnetic Resonance Study of the Hybrid Perovskites CH3NH3PbX3 ( $\mathrm{X}=\mathrm{I}, \mathrm{Br}$ and $\mathrm{Cl})$. I. Mater. Chem. A 2015, 3, 9298-9307.

(35) Hua, G. L. Normal Vibration Modes and the Structural Phase Transitions in Caesium Trichloroplumbate $\mathrm{CsPbCl} \mathrm{3.} \mathrm{L.} \mathrm{Phys.:}$ Condens. Matter 1991, 3, 1371.

(36) Even, J.; Carignano, M.; Katan, C. Molecular Disorder and Translation/Rotation Coupling in the Plastic Crystal Phase of Hybrid Perovskites. Nanoscale 2016, 8, 6222-6236.

(37) Fujii, Y.; Hoshino, S.; Yamada, Y.; Shirane, G. NeutronScattering Study on Phase Transitions of $\mathrm{CsPb} C l$ 3. Phys. Rev. B 1974, 9, 4549-4559.

(38) Harris, M. J.; Cowley, R. A.; Swainson, I. P.; Dove, M. T. Observation of Lattice Melting at the Ferroelastic Phase Transition in Na2CO3. Phvs. Rev. Lett. 1993, 71, 2939-2942.

(39) Even, J. Pedestrian Guide to Symmetry Properties of the Reference Cubic Structure of 3D All-Inorganic and Hybrid Perovskites. I. Phys. Chem. Lett. 2015, 6, 2238-2242.
(40) Chen, T.; Foley, B. J.; Ipek, B.; Tyagi, M.; Copley, J. R. D.; Brown, C. M.; Choi, J. J.; Lee, S.-H. Rotational Dynamics of Organic Cations in the CH3NH3PbI3 Perovskite. Phvs. Chem. Chem. Phvs. 2015, 17, 31278-31286.

(41) Leguy, A. M. A.; Frost, J. M.; McMahon, A. P.; Sakai, V. G.; Kochelmann, W.; Law, C.; Li, X.; Foglia, F.; Walsh, A.; O’Regan, B. C.; et al. The Dynamics of Methylammonium Ions in Hybrid OrganicInorganic Perovskite Solar Cells. Nat. Commun. 2015, 6, 7124.

(42) Fabini, D. H.; Hogan, T.; Evans, H. A.; Stoumpos, C. C.; Kanatzidis, M. G.; Seshadri, R. Dielectric and Thermodynamic Signatures of Low-Temperature Glassy Dynamics in the Hybrid Perovskites $\mathrm{CH} 3 \mathrm{NH} 3 \mathrm{PbI} 3$ and $\mathrm{HC}(\mathrm{NH} 2) 2 \mathrm{PbI} 3$. I. Phvs. Chem. Lett. 2016, 7, 376-381.

(43) Park, J.-S.; Choi, S.; Yan, Y.; Yang, Y.; Luther, J. M.; Wei, S.-H.; Parilla, P.; Zhu, K. Electronic Structure and Optical Properties of $\alpha$ -

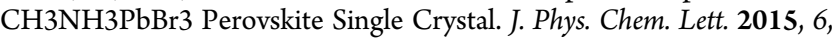
4304-4308.

(44) Brittman, S.; Garnett, E. C. Measuring N and $\mathrm{K}$ at the Microscale in Single Crystals of $\mathrm{CH} 3 \mathrm{NH} 3 \mathrm{PbBr} 3$ Perovskite. I. Phvs. Chem. C 2016, 120, 616-620.

(45) Feng, J. Mechanical Properties of Hybrid Organic-Inorganic $\mathrm{CH}_{3} \mathrm{NH}_{3} \mathrm{BX}_{3}(\mathrm{~B}=\mathrm{Sn}, \mathrm{Pb} ; \mathrm{X}=\mathrm{Br}, \mathrm{I})$ Perovskites for Solar Cell Absorbers. APL Mater. 2014, 2, 081801.

(46) Piskunov, S.; Heifets, E.; Eglitis, R. I.; Borstel, G. Bulk Properties and Electronic Structure of $\mathrm{SrTiO}_{3}, \mathrm{BaTiO}_{3}, \mathrm{PbTiO}_{3}$ Perovskites: An Ab Initio HF/DFT Study. Comput. Mater. Sci. 2004, 29, 165-178.

(47) Rakita, Y.; Cohen, S. R.; Kedem, N. K.; Hodes, G.; Cahen, D. Mechanical Properties of APbX 3 (A = Cs or $\mathrm{CH} 3 \mathrm{NH} 3$; X $=\mathrm{I}$ or $\mathrm{Br}$ ) Perovskite Single Crystals. MRS Commun. 2015, 5, 623-629.

(48) Shabbir, G.; Kojima, S. Central Peak and Acoustic Anomalies of the Relaxor Ferroelectric Lead Magnesium Niobate-Lead Titanate Single Crystal Studied by the Micro-Brillouin Scattering. Appl. Phys. Lett. 2007, 91, 062911.

(49) Saidaminov, M. I.; Abdelhady, A. L.; Murali, B.; Alarousu, E.; Burlakov, V. M.; Peng, W.; Dursun, I.; Wang, L.; He, Y.; Maculan, G.; et al. High-Quality Bulk Hybrid Perovskite Single Crystals within Minutes by Inverse Temperature Crystallization. Nat. Commun. 2015, 6,7586 .

(50) Ollivier, J.; Ecolivet, C.; Beaufils, S.; Guillaume, F.; Breczewski, T. Light Scattering by Low-Frequency Excitations in Quasi-Periodic N-Alkane/Urea Adducts. EPL Europhvs. Lett. 1998, 43, 546.

(51) Sandercock, J. R. Trends in Brillouin Scattering: Studies of Opaque Materials, Supported Films, and Central Modes. In Light Scattering in Solids III; Cardona, M., Güntherodt, G., Eds., Springer: Berlin, 2014.

(52) Toudic, B.; Lefort, R.; Ecolivet, C.; Guérin, L.; Currat, R.; Bourges, P.; Breczewski, T. Mixed Acoustic Phonons and Phase Modes in an Aperiodic Composite Crystal. Phvs. Rev. Lett. 2011, 107, 205502. 